\title{
Detecting nonlinearity in run-up on a natural beach
}

\author{
K. R. Bryan ${ }^{1}$ and G. Coco ${ }^{2}$ \\ ${ }^{1}$ Department of Earth and Ocean Sciences, University of Waikato, Private Bag 3105, Hamilton, 3240, New Zealand \\ ${ }^{2}$ National Institute of Water and Atmospheric Research, P.O. Box 11-115, Hamilton, New Zealand
}

Received: 8 March 2007 - Revised: 8 June 2007 - Accepted: 11 June 2007 - Published: 12 July 2007

\begin{abstract}
Natural geophysical timeseries bear the signature of a number of complex, possibly inseparable, and generally unknown combination of linear, stable non-linear and chaotic processes. Quantifying the relative contribution of, in particular, the non-linear components will allow improved modelling and prediction of natural systems, or at least define some limitations on predictability. However, difficulties arise; for example, in cases where the series are naturally cyclic (e.g. water waves), it is most unclear how this cyclic behaviour impacts on the techniques commonly used to detect the nonlinear behaviour in other fields. Here a non-linear autoregressive forecasting technique which has had success in demonstrating nonlinearity in non-cyclical geophysical timeseries, is applied to a timeseries generated by videoing the waterline on a natural beach (run-up), which has some irregular oscillatory behaviour that is in part induced by the incoming wave field. In such cases, the deterministic shape of each run-up cycle has a strong influence on forecasting results, causing questionable results at small (within a cycle) prediction distances. However, the technique can clearly differentiate between random surrogate series and natural timeseries at larger prediction distances (greater than one cycle). Therefore it was possible to clearly identify nonlinearity in the relationship between observed run-up cycles in that a local autoregressive model was more adept at predicting run-up cycles than a global one. Results suggest that despite forcing from waves impacting on the beach, each run-up cycle evolves somewhat independently, depending on a non-linear interaction with previous run-up cycles. More generally, a key outcome of the study is that oscillatory data provide a similar challenge to differentiating chaotic signals from correlated noise in that the deterministic shape causes an additional source of autocorrelation which in turn influences the predictability at small forecasting distances.
\end{abstract}

Correspondence to: K. R. Bryan

(k.bryan@waikato.ac.nz)

\section{Introduction}

Most timeseries collected from natural systems are to some degree difficult to predict. The possibility that this property is a signature of low-dimensional chaotic underlying dynamics has sparked a keen interest in exploring non-linear techniques for data analysis, model development and prediction across a wide range of fields. Early attempts in nearshore oceanography centred on direct applications of techniques developed primarily for theoretical chaotic signals, for example applying fractal or correlation dimensions (e.g. Grassberger and Procaccia, 1983) to beach morphology measurements (Southgate and Möller, 2000) or wave timeseries (Elgar and Mayer-Kress, 1989) or calculating the Lyapunov exponents of ocean water levels (Frison et al., 1999). However, there is a growing body of literature that shows these techniques do not generally work for the stochastic timeseries characteristic of natural systems, because they are very sensitive to, for example, noise, pre-processing, sampling strategy and timeseries length (Shirer et al., 1997, give a review). The key issue is that natural systems, even if they have a chaotic element, do not evolve in isolation but receive stochastic forcing from external sources. The techniques that have been developed for theoretical chaotic signals are not equipped to deal with such mixed and noisy processes, and, dangerously, often provide spurious results. For example, synthetic linear timeseries with random noise added can provide equally strong "evidence" of chaotic behaviour in ocean gravity waves (Elgar and Mayer-Kress, 1989), beach profile behaviour (Elgar, 2001) and in radar measurements of the ocean surface (sea clutter) (Haykin et al., 2002).

As with research in the marine environment generally (e.g. Hsieh et al., 2005), these kinds of problems have sadly deterred exploration of chaotic behaviour in nearshore oceanography particularly in environments that are already known to comprise a mixture of many different processes. For example, there are few published results that have

Published by Copernicus Publications on behalf of the European Geosciences Union and the American Geophysical Union. 
successfully demonstrated chaotic behaviour in common signals such as timeseries of gravity waves, seabed ripples, beach morphology and run-up (run-up is the movement of the waterline on a beach). In fact, to what degree this chaotic behaviour emerges in natural systems is not clear.

The case of run-up is particularly interesting because it encompasses a feedback behaviour which clearly has the potential of inducing a chaotic response. As a wave crest enters the run-up zone, it progresses up the beachface as a thinning tongue of water ("uprush") until the influence of gravity causes a reversal of flow direction. The water flows back down the beachface under the influence of gravity ("downrush"). However, depending on the size of the wave, on the timing between successive wave crests and the beach slope, the downcoming water can interact, sometimes violently with the incoming water, removing momentum from the new incoming wave. Alternatively, the incoming wave can join the uprush of the previous wave and produce an extra large run-up event. So the process is complex in that it is some unknown combination of forcing from the incoming wave field and dynamics which evolve within the run-up zone. The mismatch between the timing of the forcing and run-up cycle is critical to the dynamics. Such a system is reminiscent of some of the classic examples of chaotic behaviour such as the ball bouncing on an oscillating plate or a kicked rotor (see Tuffillaro and Albano, 1986; or Moon, 2005 , for a review). In such an analogy, the run-up cycles represent the bouncing ball or the rotor and the forcing caused by the incoming waves represents the oscillating plate or the "kick". Although intriguing, there is no evidence to prove or disprove the existence of chaotic behaviour in run-up.

Natural signals that are potentially chaotic require different analysis approaches because they represent a combination of a deterministic chaotic signal combined with measurement and dynamical noise. Measurement noise is simply superimposed on the system and thus techniques aim to simply extract the signal from the noise. Conversely, dynamical noise can force the deterministic nonlinear portion of the signal to occupy different states (e.g. stable or non-stable) and thus techniques for detecting stochastic chaos must be developed with regard to this inherent inseparability (Sugihara, 1994).

Casdagli (1989) and later Sugihara and May (1990) apply a nonlinear autoregressive forecasting technique developed by Farmer and Sidorowich (1987) as a tool for detecting nonlinearity in stochastically-forced non-linear signals. Casdagli (1992) used the relative difference in performance of models with differing numbers of nearest neighbours $k$ included in the model as an indicator of nonlinearity, where superior performance at larger $k$ (global model) indicates a linear underlying process and superior performance at smaller $k$ (local model) indicates that the modelled timeseries exhibits local behaviour and thus the underlying process is more likely to be chaotic. Intermediate $k$ is an indication of stochastic non- linearity (Sugihara, 1994). The behaviour of the correlation between forecast and observations with increasing prediction time (e.g. Sugihara and May, 1990) can be used to differentiate between stable and unstable nonlinearity (or chaos), with unstable correlation coefficients decaying exponentially and more quickly with time (Sugihara, 1994).

This nonlinear forecasting technique has successfully demonstrated local behaviour in natural systems such as phytoplankton dynamics, electrical heart rate signals (both in Sugihara, 1994), sediment transport (Jaffe and Rubin, 1996), airborne cedar pollen timeseries (Delaunay et al., 2004), thermoregulatory responses in plants (Ito and Ito, 2005), fisheries timeseries (fish landings, larval abundance (Hsieh et al., 2002)) and photos of wind ripples (Rubin, 1992). However, often global autoregressive models seem more adept at predicting natural geophysical timeseries such as sea surface temperature signals (Hsieh et al., 2005), geomagnetic timeseries associated with volcanic activity (Currenti et al., 2004) and eruptive activity of a volcano (Marzocchi et al., 1997) and electrical precursory timeseries used for earthquake prediction (Cuomo et al., 1998) possibly because of the levels of dynamical noise associated with these signals. Such failures have lead to conclusions that "evidence of chaos in geophysical timeseries does not seem statistically significant" (p. 3207 in Marzocchi et al., 2004).

Here we apply the same non-linear forecasting technique to timeseries generated from digitizing video footage of the water's edge (the run-up) on a beach as it moves up and down the beachface with each incoming wave. Such a timeseries provides an interesting challenge, as it encompasses a moreor less deterministic shape (the parabola traced by the run-up as it moves up and down the beachface) appended together in a timeseries, with each parabola related to each other in some unknown way depending on stochastic forcing from the incoming wave field and interactions between run-up events. The nonlinearity of interest here is not the nonlinear shape of the parabola followed by each run-up cycle (which is not a signature of dynamic nonlinearity), but the nonlinearity associated with interactions between run-up events, which we believe has the potential to be unstable and therefore exhibiting chaotic behaviour.

\section{Methods}

\subsection{Description of non-linear forecasting technique}

In a linear or global autoregressive model every point in a time series $x(t)$ is regressed against the previous sequence of points $x(t-j \Delta t)$ to evaluate the coefficients $a_{j}$ in

$x(t+d \Delta t)=a_{o}+\sum_{j=0}^{j=m-1} a_{j+1} x(t-j \Delta t)$

where $\Delta t$ is the time lag between points in the sequence, $d$ represents distance into the future and $m$ is the number 

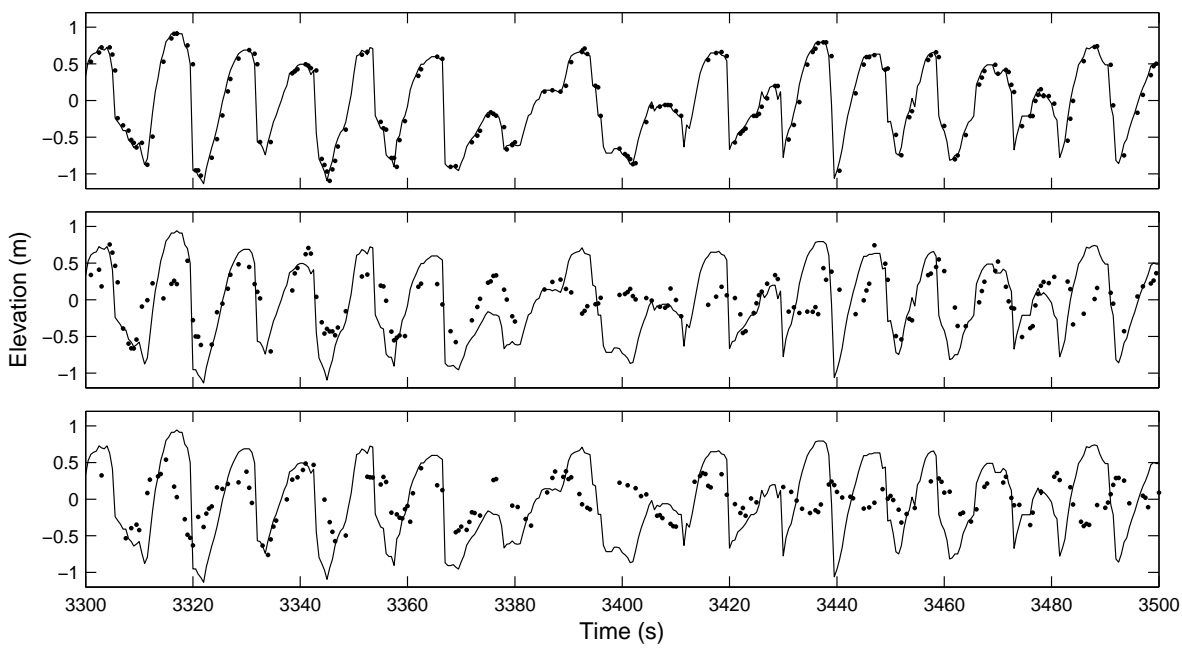

Fig. 1. (a) An example of the run-up timeseries. Bullets indicate the points for which a forecast is made. (b) The $d=\Delta t$ (=3.5 $\mathrm{s}$ ) forecast using $m=7$ and $k=500$. The line indicates the original series and the bullets represent the points for which a forecast is made. (c) The $d=5 \Delta t$ forecast for the same data.

of coefficients. This time lag can be evaluated using the first minimum in the average mutual information (e.g. Abarbanel, 1986), which is a measure of the information gained about one measurement using another. To evaluate the performance of the model, timeseries can be divided into training and testing series where predictions for the testing series are made using the coefficients $a_{j}$ that are evaluated by using all available sequences from the training dataset. The prediction can be evaluated by either the correlation (e.g. Sugihara and May, 1990) or the root-mean-square error (e.g. Farmer and Sidorowich, 1987) between prediction and observation.

In a non-linear or local autoregressive model, the coefficients $a_{j}$ are only evaluated using training sequences which are nearest neighbours to the testing sequence for which a prediction is required. Therefore the model can be tuned to local behaviour and therefore can provide improved predictions for non-linear processes. In the linear model, the system evolves in the same way regardless of initial conditions, so maximizing the number of nearest neighbours $k$ will give the best model because this will minimize the noise component most effectively. As a consequence, it is this difference in predictive ability between the global $(k=n$, where $n$ is the total number of available sequences) and local model $(k<n)$ that allows the autoregressive model to be used as a tool to explore the underlying nature of the process generating the timeseries. In this case the placket size (or the number of points in the sequences), $m$, is analogous to the embedding dimension of the non-linear system. The ability of the technique to perform in cases where timeseries lengths are short and contaminated by moderate levels of noise make the technique more appropriate for analysis of timeseries from natural systems than tools such as the fractal dimension, correlation dimension and the Lyapunov exponents that have been developed for deterministic chaotic signals. However, when the level of measurement noise increases significantly, the linear model provides a better prediction regardless of the underlying nature of the timeseries (Rubin, 1995).

\subsection{Run-up data collection and analysis}

The timeseries of run-up (e.g. Fig. 1a) used here were digitized from sub-aerial video imagery collected at the US Army Corps of Engineers Field Research Facility at Duck North Carolina, on 6 September 1994 (these timeseries have been used previously in Burnet (1998) and Ciriano et al. (2005). In addition, Coco et al. (2003) provide details on the methods used to extract run-up timeseries from video). Timeseries were collected at $2 \mathrm{~Hz}$ and converted to variations in water level elevation with the help of surveyed measurements of beachface morphology and standard camera rectification techniques. The observations reported here were collected during a day where the incoming waves were from a narrow range of directions and periods, with a peak spectral period of $12 \mathrm{~s}$. Above the main peak, the spectrum decayed at $\sim f^{-3}$, where $f$ is frequency, which is similar to the theoretical spectral signature of a timeseries of parabolas $\left(\sim f^{-4}\right.$ (Mase, 1988)). In the high frequency region of the spectrum of run-up, the energy remained constant as frequency increased, indicating that the signal was below the measurement capabilities of the video technique. This was assumed to be the measurement noise component of the signal and $\left(v_{\text {noise }}\right)^{0.5}$ equated to $<9 \%$ of the $\left(v_{\text {signal }}\right)^{0.5}$, where $v_{\text {noise }}$ and $v_{\text {signal }}$ are the summed variance associated with the noise and the run-up signal in the spectrum respectively.

To implement the forecasting technique, a randomlyselected testing sequence of length $m+d \Delta t$ was removed from the original detrended 14000 point run-up timeseries, 


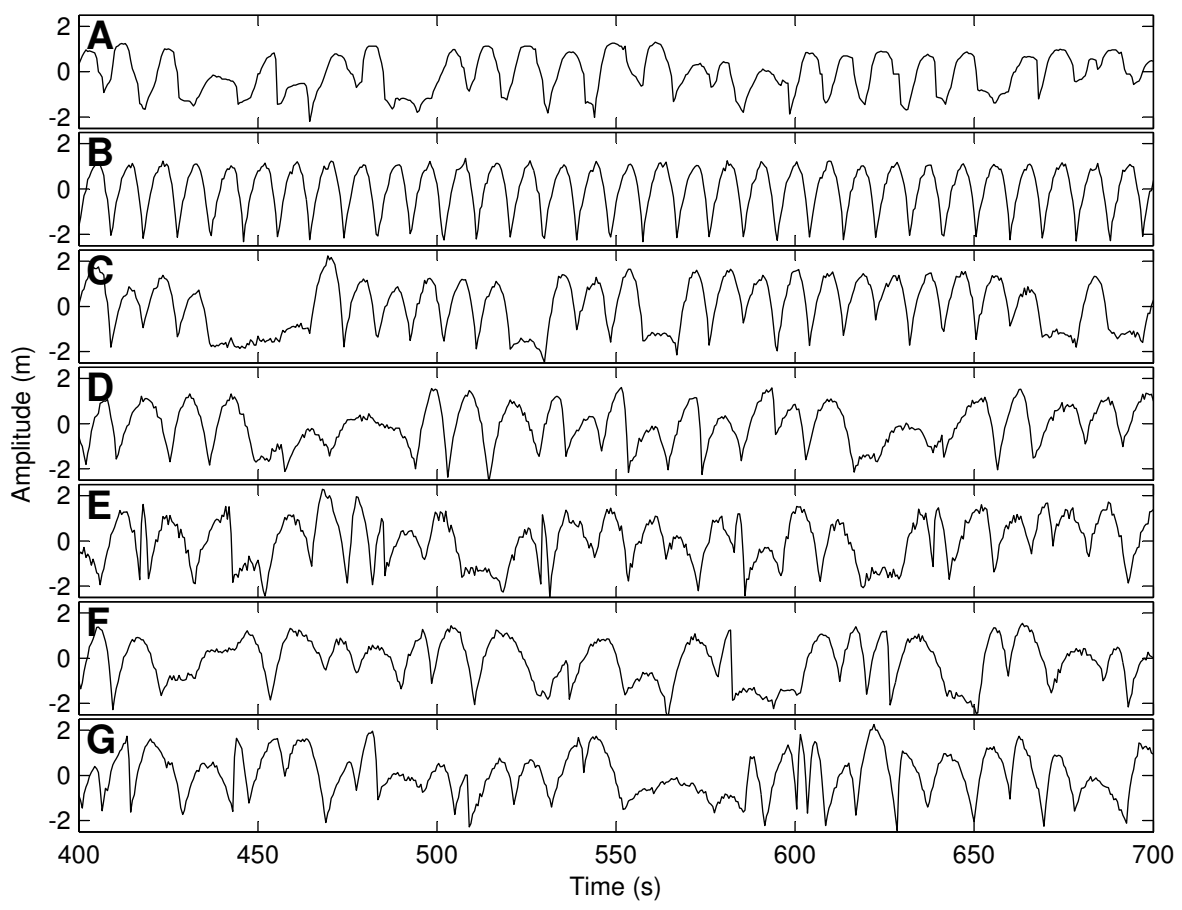

Fig. 2. (A) The natural timeseries. (B) The surrogate created by appending parabolas with using $S_{\max }, S_{\min }, d T, T$ equal to the mean of the values in library created by measuring these values in the natural series. (C) The surrogate created using $S_{\max }$ and $S_{\min }$ selected randomly from the library, but $d T$ and $T$ set equal to the mean of available library values. (D) $S_{\max }$, and $T$ all selected randomly from the library. (E) $S_{\min }, d T$, and $T$ given the same order as in the natural series, $S_{\max }$ selected randomly from the library. (F) $S_{\max }, S_{\min }$, and $d T$ given the same order as in the natural series, $T$ selected randomly from the library. (G) $S_{\max }, S_{\min }, d T, T$ selected randomly from the library, and a sinusoid with amplitude of 0.2 and period of 20 s superimposed.

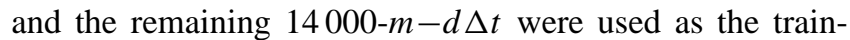
ing data set. This method was implemented to maximize the training dataset, since smaller training datasets (although computationally advantageous) appeared to provide more variable results. The regression coefficients (and the selection of nearest neighbours) were re-evaluated for each step into the future $d$. This process was repeated 6000 times (insuring each testing sequence was only included once) to allow a robust estimate of the correlation between prediction and observation (smaller numbers of repetition resulted in more variable results). The analysis was then repeated to optimize the number of nearest neighbours $k$ and the placket size $m$. Significance levels on the resulting correlations were calculated by randomising the original timeseries, and repeating the analysis 100 times using 500 test cases each time (rather than the computationally-intensive 6000 test cases).

\subsection{Surrogate series}

One of the difficulties in interpreting the results is isolating the influence of the somewhat deterministic parabolic shape of each run-up event on the forecasting technique. To address this, a number of surrogate series were created to mimic timeseries of naturally occurring surf-zone processes, using similar methods to Theiler et al. (1992). The difficulty is cre- ating a surrogate series that is random, yet with many of the properties of the original series (Small and Tse, 2002). In our case the challenge is to preserve shape that is characteristic of each run-up event and the gross statistical properties of all the events. The water level during each run-up cycle does not return to a common level, therefore the shuffling scheme used by Theiler (1995) and Stone (1992) needed adaptation. To this end, the height and timing of the maximum and minimum of each run-up cycle was extracted from the natural timeseries and used to create a library of $\sim 760$ run-up maxima $S_{\max }$, run-up minima $S_{\min }$, the time separation of consecutive run-up maxima $T$, and the time separation of consecutive run-up maxima and minima $d T$. Synthetic series were created by randomnly selecting characteristics from the library, and fitting two half parabolas for each run-up cycle: between the minimum and maximum, and between the maximum and the following minimum. The selections from the library required a limitation in that $S_{\max }>S_{\min }$ and $d T<T$. However, in practice these cases were rare. In this way, the resulting run-up series had the same statistical distribution of $S_{\max }, S_{\min }, d T, T$, but different order.

A number of synthetic series were created using this technique, ranging from using the original sequence of parameters (so that the resulting series was very similar to the data 
which is shown in Fig. 2a, using constant values for the parameters (the averages) (Fig. 2b), randomly selecting $S_{\max }$ and $T$, but using the $S_{\min }$ and $d T$ associated with that $S_{\max }$ so that the shape of the leading face of each run-up event is preserved exactly (Fig. 2d), and combinations thereof (Figs. 2c, e and f). It must be noted, that in cases in which all 4 parameters are randomly selected independently from the library (not shown), the resulting timeseries appeared quite different from the original because the joint probability distributions are not preserved. Finally, relationships between run-up cycles were added to the random surrogate series, for example $S_{\max }$ was related to the previous $S_{\max }$, or the previous $S_{\max }-S_{\min }$, or a low frequency sinusoid added (Fig. $2 \mathrm{~g}$ ), to mimic low frequency energy generally observed in run-up timeseries (the data used here included). All series were detrended and standardised and normally distributed random noise with 0.09 standard deviation superimposed. Many more variations were trialled than presented in Fig. 2.

\section{Results}

\subsection{Run-up on a natural beach}

The predictions (examples given in Figs. 1b and c) capture the cyclic nature of the observations, albeit an under prediction of maxima and an overprediction of minima which worsens with increasing $d$. Interesting behaviour occurs around $t=3380 \mathrm{~s}$ where the uprush event of several run-up cycles merge to result in an extra high and long run-up cycle. The prediction at $d=\Delta t(=3.5 \mathrm{~s})$ does reproduce some of this low frequency behaviour, although entirely misses the minima at $3400 \mathrm{~s}$.

The time lag $\Delta t$ was calculated using the average mutual information to be 7 data points ( $3.5 \mathrm{~s}$ ), which, considering the average $T$ is $9.2 \mathrm{~s}$, is approximately a third of a typical runup cycle. The optimum placket size or the dimension of the system appears to be 6 or 7 points (Fig. 3), which means between two and three run-up cycles are needed to learn the behaviour of the system. The correlation of prediction with the observation at $d=\Delta t$ and at the optimum $m$ and $k$ is $\sim 0.64$.

The optimum number of nearest neighbours needed is $\sim 500$ which corresponds to $3.5 \%$ of the training data set (Figs. 3 and 4). The local model clearly provides a better prediction than the global model, although the enhancement in the correlation coefficient is only on the order of $15 \%$. As the prediction is stepped into the future, the correlation rapidly diminishes accompanied by an increase in the optimum numbers of nearest neighbours, so that at $d=10 \Delta t$ or $35 \mathrm{~s}$ ahead ( $\sim 4$ run-up cycles), correlation is 0.2 and $k=10000$ or $\sim 73 \%$ of the training dataset (Fig. 4).

\subsection{Surrogate time series}

The various surrogate run-up series revealed the controls of period and memory on the predictability and the optimum

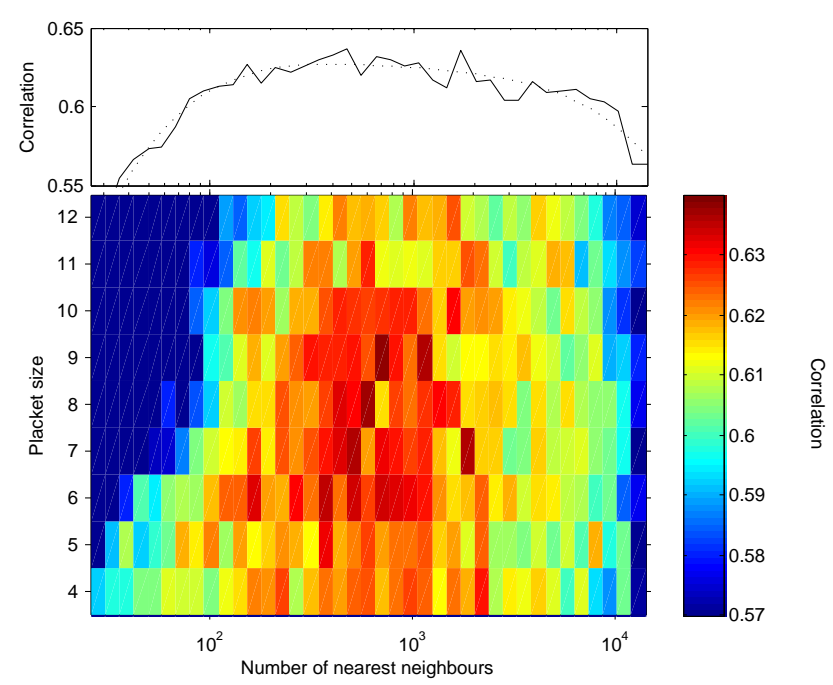

Fig. 3. Bottom panel: The greyscale represents the correlation between the forecast and the natural timeseries as a function of $k$ and $m$, at $d=\Delta t$. The correlation is evaluated by making predictions for 6000 points. Top panel: the correlation as a function of $k$ at the optimal $m$. The dotted line is the best-fit polynomial.

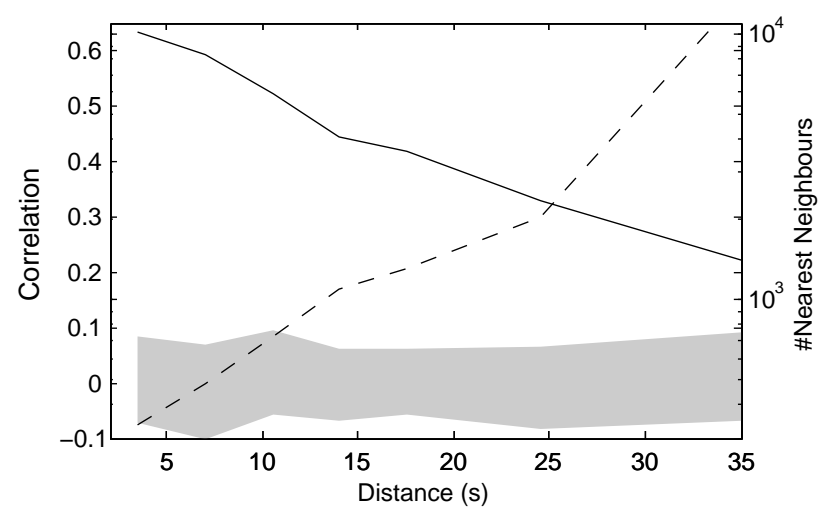

Fig. 4. Solid line: Decorrelation between forecast (using optimal $k$ and $m$ ) and natural timeseries with increasing $d$ (scale on the left). The shaded region represents the $90 \%$ confidence interval as discussed in the text. Dashed line: Increase in the optimal $k$ with increasing $d$ (scale on the right).

$k$. Examples are shown in Fig. 5. Unsurprisingly, the runup series surrogate in which the order of parameters was the same as in the observations produced nearly identical results to the actual data (not shown) which was a good check on the method of producing surrogates. Fixing all the parameters to their mean values allowed near perfect predictability (apart from the superimposed random component), with the prediction reaching a constant level at $k>300$ for all $d$, indicating that $\sim 2 \%$ of a run-up cycle is enough to learn the parabolic shape (Fig. 5, lines with crosses). There is a very slight enhancement $(\sim 0.1 \%)$ of the nonlinear over the linear model, where the former is more capable of tuning to the sharp point 

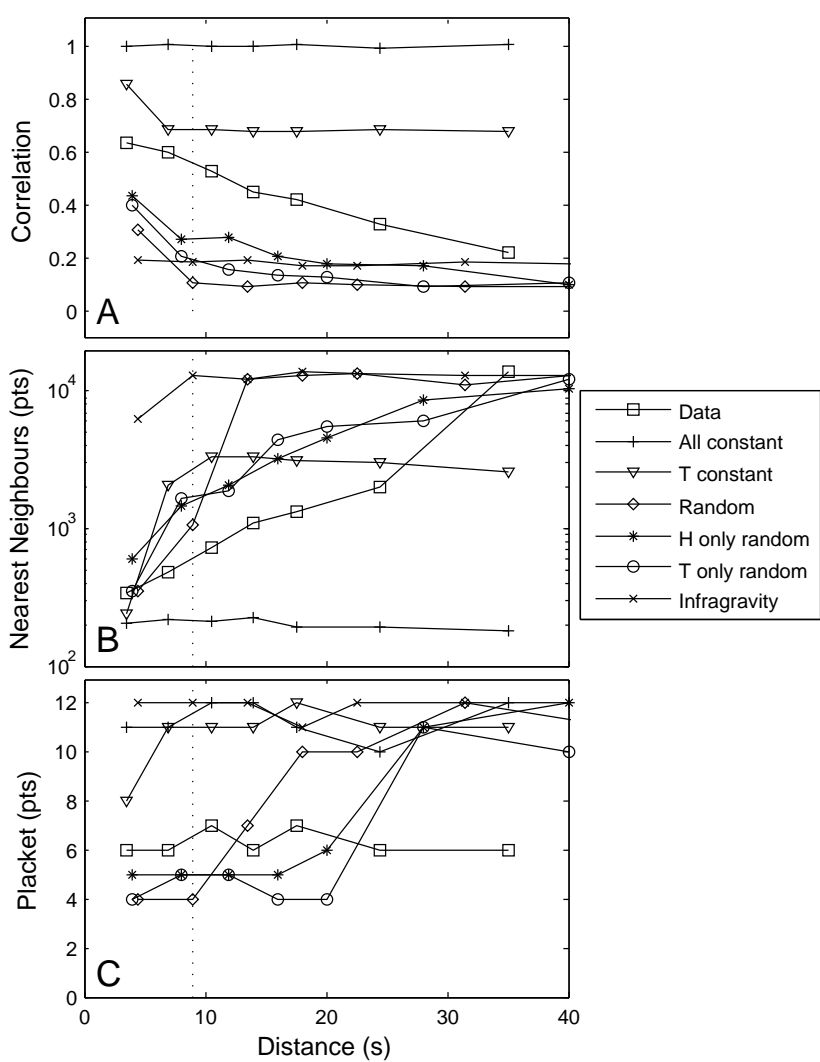

Fig. 5. (A) Correlation as a function of $d$ for the series presented in Fig. 2. (B) Optimum $k$ as a function of $d$ for the same cases. (C) Optimum $m$ as a function of $d$ for the same cases. The vertical dotted line in all panels represents the period of a typical run-up cycle.

at the join between parabolas. We tried other shapes such as a saw-tooth, sinusoid, and repeated spikes, and in all cases the smoother the shape, the better the linear model performs (albeit with only very slight differences). Larger plackets also provided better results in these cases.

In the case in which $S_{\max }$ and $T$ were chosen randomly from the library (Fig. 5, diamonds), the timeseries was completely unpredictable beyond one run-up cycle $(d>9.2 \mathrm{~s})$. Within the run-up cycle, there was a small degree of predictability (correlation $\sim 0.2$ ). The optimum $k$ appeared to be inversely related to the correlation in that within this first runup cycle, the optimum $k$ was similar to the results obtained using the natural series, and beyond the first cycle the global model was the best (maximum $k$ ). Likewise, the placket size changed from $\sim 3$ within the first run-up cycle to the maximum trialled (12 points) beyond the first run-up cycle.

Fixing $T$ only, while selecting the other variables randomly dramatically increased the predictability over the completely random case, and the predictability remained relatively constant for increasing $d$ (compare Fig. 5, triangles and diamonds). Finally, randomising $S_{\max }$ and $T$ separately while using the observed order for the other variables decreased predictability by $30 \%$ and $50 \%$ respectively both within and beyond the first run-up cycle. Interestingly, the optimum $k$ increased much more quickly with increasing $d$ for these two cases than it did for the results obtained using the natural series (Fig. 5b). For both cases with fixed $T$, the largest placket size (e.g. 12 points) provided the optimum prediction, whereas for all other cases other than the exact replica of the data, a placket of 3-4 points provided the optimum fit, increasing with increasing $d$. (One run-up cycle is approximately 3-4 points).

Finally, adding a low frequency undulation to the surrogate did not succeed in replicating the results obtained using the observations. For example, adding a low-frequency sinusoid (Fig. 5, "x") to the otherwise completely random case, served to increase the predictability beyond the first run-up cycle, and maintain this predictability at a constant level (Fig. 5, compare " $\mathrm{x}$ " and diamonds). Interestingly the optimum $k$ and $m$ increased dramatically, as in the cases using constant $T$. It is possible to manipulate the out-of-cycle predictability in the case where all four parameters are chosen randomly, by adding a (linear) memory to $S_{\max }$. However, in contrast to the results obtained using the natural timeseries, the global model (maximum $k$ ) provided the best out-of-cycle prediction in these cases and we could not reproduce the level of predictability that was obtained using the natural run-up series by using a simple linear model.

\section{Discussion and conclusions}

It is clear from the surrogate testing that the results need to be interpreted differently for predictions that are within cycle (in which the placket includes a portion of the cycle that is being predicted) and those that are out-of-cycle (in which a prediction is being made for an entirely new run-up cycle). It was always possible to make some level of prediction within-cycle, even when the parameters controlling the shape of run-up events were randomly chosen. This was because all run-up events were based on the same shape. The natural timeseries was best predicted by a local model, with $k=500$, and therefore the model is using the information from $\sim 27$ sequences of 2 run-up cycles $(=18.4 \times 2$ points) to train the model. There are a total of $\sim 760$ sequences of 2 run-up cycles in the 14000-point timeseries, so this amounts to using approximately $3.5 \%$ of the available information to train the model. When making within cycle predictions, the placket that is used includes points from the current cycle, so the model can make a successful prediction by finding a runup cycle that is evolving in a similar way from the cycles available in the training dataset. This is in contrast to the out-of-cycle predictions where the model includes no information from the cycle it is attempting to predict. Note that it is impossible to randomly order $S_{\max }$ and $T$ at the same time as creating a series of parabolas that exactly mimic the 
shape of run-up cycles in the data, and this is why the surrogate in which the cycles are randomised (Fig. 5a, diamonds) has considerably reduced within-cycle correlation relative to results based on the natural timeseries.

Although the forecasting technique can successfully identify the parabolic shape as nonlinear because the within-cycle behaviour is best predicted by a local model, as stated previously, this parabolic shape can be modelled with a linear dynamical equation and so is unlikely to be an indication of dynamic nonlinearity. There do exist weakly non-linear terms in, for example, the effect of bottom friction, which play a role in the evolution of the run-up cycle. However, given the results of the surrogate testing, the "nonlinearity" identified by studying the behaviour of the within-cycle forecasts is not of interest in our goal of understanding the complex dynamics of run-up cycles. For example, the surrogate series with randomly appended parabolas was best fit (for withincycle predictions) with the local model with the optimum $k$ the same as for the natural timeseries, even though such a series is completely synthetic and clearly does not represent underlying chaotic dynamics. With 760 synthetic run-up cycles with which to train the model, many combinations of 2 run-up cycles are represented in the training dataset, and the local model is more adept to tuning to these shapes. $\mathrm{Ru}$ bin (1992) discuss a similar case, in which a deterministic pattern is randomly repeated, which mimicked some of the gross properties of a chaotic signal in forecasting analysis. This is probably similar to the difficulties encountered in distinguishing chaotic series from coloured noise (Tsonis and Elsner, 1992). We can therefore conclude that, within cycle, the out-performance of the non-linear model over the linear model is not a good indicator of dynamic non-linearity in the timeseries because it cannot differentiate between the surrogate and the natural timeseries.

Conversely, out-of cycle forecasts for the natural timeseries are clearly different than the results obtained using the surrogate where properties are randomly ordered (compare squares and diamonds in Fig. 5). In the latter case, the forecast and surrogate timeseries are essentially uncorrelated and the optimum $k$ and $m$ are maximised, whereas the correlation decays for the natural timeseries, along with a slow increase in $k$ accompanying this decorrelation. Unlike in $\mathrm{Ru}-$ bin (1992), increasing embedding dimension (packet size $m$ ) does not improve the performance of the linear over the nonlinear model in forecasting our natural run-up series (Fig. 2). Therefore, although the forecasting technique cannot distinguish dynamically nonlinear and randomly-generated signals at within-cycle forecasting distances, beyond the cycle the technique has clearly shown that run-up is non-linear in a dynamical sense. It is possible to predict to some degree a run-up cycle using only information from previous run-up cycles, and that prediction is better done with a local model.

Placket size appeared to provide some indication of the nature of the series. A placket size of approximately two run-up cycles provides the best prediction for the natural timeseries, whereas the largest placket size tested provided the best prediction for the surrogates in which period was held constant. In the case of the linear timeseries, the more data that can be included in the model (through larger $m$ and $k$ ), the more the random noise component can be suppressed and the better the fit (Fig. 5, bottom panel, lines with crosses and "x"s). In the surrogates that remove the effect of memory by randomising $S_{\max }, S_{\min }, T$ and $d T$, the optimum placket size is reduced to less than one run-up cycle (Fig. 5, bottom panel, lines with diamonds, stars and circles). When there is no ability to predict the next run-up cycle, the model then optimised the prediction within a run-up cycle $(d<3)$ and only a few points are needed for the model to learn how a run-up cycle will evolve.

Given the change in behaviour between $d=2(7 \mathrm{~s})$ and $d=3$ $(10.5 \mathrm{~s})$ caused by the differing behaviour of within and outof-cycle forecasts, it is difficult to conclude whether or not the forecast and natural timeseries decorrelate exponentially as expected for chaotic timeseries (Tsonis and Elsner, 1992). The relationship between $\log (1-r(d))$, where $r$ is the correlation, versus $d$ does display a linear scaling at prediction times beyond one run-up cycle in that there was little significant increase in fit between a line $\left(r^{2}=0.985\right.$ and a 2 nd order polynomial $\left.r^{2}=0.988\right)$. However, there are only 5 points with which to draw this conclusion.

Clearly the ability to predict the natural timeseries beyond one cycle using only information from previous cycles indicates a dependence of each run-up event on previous run-up events. The results from the randomised run-up surrogates show that this is not simply due to the run-up events being generally similar in shape. Even in the case of the surrogate in which only $S_{\max }$ is randomised, the out-of-cycle predictions are much reduced (Fig. 5, lines with stars). In the case of the natural timeseries, the evolution of each run-up cycle depends on the size and period of the bore that impacts onto the beachface, but also on the nature of the interaction of the previous run-up event. If the previous run-up event was short and small, it is like that its cycle would be complete before the next event came along. Conversely, if the run-up event was long and large, it is likely that the next uprush would interact with the downrush of the previous event. If the run-up event was very long, the two progressive uprush events would combine. In general, this run-up cycle is likely to influence the 3rd and so on. Thus the "memory" of previous run-up cycles should gradually diminish with time. Although not linear, the predictions and observations roughly decorrelate by about 0.14 per run-up cycle, and so, on average, the "memory" lasts just over 4 run-up cycles. The tests with surrogates show that making $S_{\max }$ a linear function of the previous $S_{\max }$ indicates that natural run-up series cannot be modelled with a simple linear interaction, and suggest, along with the other evidence, that processes acting in the run-up zone are inherently non-linear.

A low-frequency structure in the incoming bore such as caused by groupiness (in which there are sets of waves with 
higher wave heights) or infragravity waves (which cause a low frequency modulation of the mean water line) could also provide a source of memory in the series. It is interesting to note that the series with randomly appended parabolas has a low frequency spectral signal, which is generated when the timing between parabolas is such that the "water-line" does not return to the same level between parabolas (Fig. 2d). This is present in natural run-up series, and indeed has been some of the evidence provided for the existence of surf-zone infragravity waves. The tests with surrogate series (Fig. 5, lines with " $x$ ") indicate that a low-frequency signal in the timeseries would create a completely different type of result in which a maximum $k$ and $m$ are optimum and the correlation is fairly constant with $d$, similar to the case in which $T$ is constant. The source of this low frequency signal appears not to be a linear infragravity wave.

Despite the confounding effect of the roughly-parabolic shape of run-up cycles on the with-in cycle forecasts, we can confidently conclude using the out-of-cycle forecasts that the run-up timeseries we have measured here is better modelled with a local autoregressive than a global autoregressive model, and thus, using the practical definition of non-linear provided by Sugihara (1994), is dynamically nonlinear. However, whether the non-linear relationship between run-up cycles identified here is stable or unstable is harder to determine conclusively. An obvious next step is to use the same technique to investigate the nature of the timeseries of waves entering the run-up zone (the forcing).

Acknowledgements. Field data collection was led by B. T. Werner and S. Elgar and supported by the Office of Naval Research (USA). G. Coco was supported by the (New Zealand) Foundation for Research, Science and Technology (contract C01X0401). Work completed in part while KRB was on sabbatical leave at the University of Plymouth. Helpful comments were provided by D. A. Huntley.

Edited by: A. Baas

Reviewed by: two anonymous referees

\section{References}

Abarbanel, H. D. I.: Analysis of Observed Chaotic Data, Institute for Nonlinear Science, Springer, New York, 1986.

Burnet, T. K.: Field Testing two Beach Cusp Formation Models, Ph.D. thesis, Duke University, 1998.

Casdagli, M.: Nonlinear prediction of chaotic time series, Physica D, 35, 335-356, 1989.

Casdagli, M.: Chaos and deterministic versus stochastic non-linear modelling, J. R. Statist. Soc. B, 54, 303-328, 1992.

Ciriano, Y. Coco, G. Bryan, K. R., and Elgar, S.: Field observations of swash zone infragravity motions and beach cusp evolution, J. Geophys. Res. 110, C02018, 1-10, 2005.

Coco, G., Burnet, R. K., Werner, B. T., and Elgar, S.: Test of selforganization in beach cusp formation J. Geophys. Res., 108(C3), 3101, 1-11, 2003.
Cuomo, V., Lapenna, V., Macchiato, M., Serio, C., and Telesca, L.: Linear and nonlinear dynamics in electrical precursory time series: implications for earthquake prediction, Tectonophysics, 287, 279-298, 1998.

Currenti, G., Del Negro, C., Fortuna, L., Napoli, R., and Vicari, A.: Non-linear analysis of geomagnetic time series from Etna volcano, Nonlin. Processes Geophys., 11, 119-125, 2004, http://www.nonlin-processes-geophys.net/11/119/2004/.

Delaunay, J. J., Seymour, C., and Fouillet V.: Investigation of shortrange cedar pollen forecasting, Phys. Rev. E, 70, 066214, 1-9, 2004.

Elgar, S.: Coastal profile evolution at Duck, North Carolina: A cautionary note, J. Geophys. Res., 106(C3), 4625-4628, 2001.

Elgar, S. and Mayer-Kress, G.: Observations of the fractal dimension of deep- and shallow-water ocean surface waves, Physica D, 37, 104-108, 1989.

Farmer, J. D. and Sidorowich, J. J.: Predicting chaotic time series, Phys. Rev. Lett., 59, 84-848, 1987.

Frison, T. W., Abarbanel, H. D. I., Earle, M. D., Schultz, J. R., and Scherer, W. D.: Chaos and predictability in ocean water levels, J. Geophys. Res. 104(C4), 7935-7951, 1999.

Grassberger, P. and Procaccia, I.: Characterization of strange attractors, Phys. Rev. Lett., 50, 346-349, 1983.

Haykin, S., Bakker, R., and Currie, B. : Uncovering nonlinear dynamics: the case study of sea clutter, Proceedings of the IEEE, Special Issue on Applications of Nonlinear Dynamics to Electronic and Information Engineering, 90(5), 860-881, 2002.

Hsieh, C., Glaser, S. M., Lucas, A. J., and Sugihara, G.: Distinguishing random environmental fluctuations from ecological catastrophes for the North Pacific Ocean, Nature, Lond., 435, 336-340, 2005.

Ito, T. and Ito, K.: Nonlinear dynamics of the homeothermic temperature control in skunk cabbage, Symplocarpus foetidus, Phys. Rev. E, 72, 051909, 1-6, 2005.

Jaffe, B. E. and Rubin, D. M.: Using nonlinear forecasting to learn the magnitude and phasing of time-varying sediment suspension in the surf zone, J. Geophys. Res., 101(C6), 14 283-14296, 1996.

Marzocchi, W., Mulargia, F., and Gonzato, G.: Detecting lowdimensional chaos in geophysical time series, J. Geophys. Res., 102(B2), 3195-3209, 1997.

Mase, H.: Spectral characteristics of random wave run-up, Coast. Eng., 12, 175-189, 1988.

Moon, F. C.: Chaotic Vibrations, John Wiley and Sons, 309 pp., 2005.

Rubin, D. M.: Use of forecasting signatures to help distinguish periodicity, randomness, and chaos in ripples and other spatial patterns, Chaos 2, 525-535, 1992.

Rubin, D. M.: Forecasting techniques, underlying physics and applications, in: Nonlinear Dynamics and Fractals: New Numerical Techniques for Sedimentary Data, edited by: Middleton, G. V., Plotnick, R. E., and Rubin, D. M., SEPM Short Course No. 36., Society for Sedimentary Geology, 174 pp., 1995.

Shirer, H. N., Fosmire, C. J., Wells, R., and Suciu, L.: Estimating the correlation dimension of atmospheric time series, J. Atmos. Sci., 54, 211-229, 1997.

Small, M. and Tse, C. K.: Applying the method of surrogate data to cyclic timeseries, Physica D, 164, 18-201, 2002.

Southgate, H. N. and Möller, I.: Fractal properties of coastal profile 
evolution at Duck, North Carolina, J. Geophys. Res., 105(C5), 11 489-11 508, 2000.

Stone, L.: Coloured noise or low-dimensional chaos, Proc. R. Soc. Lond. B, 250, 77-81, 1992.

Sugihara, G.: Nonlinear forecasting for the classification of natural time series, Phil. Trans. R. Soc. Lond. A, 348, 447-495, 1994.

Sugihara, G. and May, R.: Nonlinear forecasting as a way of distinguishing chaos from measurement error in time series, Nature, Lond., 344, 734-741, 1990.

Theiler, J.: On the evidence for low-dimensional chaos in an epileptic electroencephalogram, Physics A, 196, 335-341, 1995.
Theiler, J., Eubank, S., Longtin, A., Galdrikian, B., and Farmer, J. D.: Testing for nonlinearity in time series: the method of surrogate data, Physica D, 58, 77-94, 1992.

Tsonis, A. A. and Elsner, J. B.:Nonlinear prediction as a way of distinguishing chaos from random fractal sequences, Nature, 358, 6383, 217-220, 1992.

Tuffilaro, N. B. and Albano, A. M.: Chaotic dynamics of a bouncing ball, Am. J. Phys., 54(10), 939—944, 1986. 